\title{
BENEFÍCIOS E EFEITOS DO MÉTODO PILATES NO TRATAMENTO DE DOR LOMBAR CRÔNICA INESPECÍFICA: UMA REVISÃO INTEGRATIVA
}

\section{BENEFITS AND EFFECTS OF THE PILATES METHOD IN THE TREATMENT OF NONSPECIFIC CHRONIC LOW BACK PAIN: AN INTEGRATIVE REVIEW}

Maria Izabel Alves de Carvalho ${ }^{1}$, Lorrane Caroline de Oliveira ${ }^{2}$

\section{RESUMO}

Atualmente a dor lombar crônica acomete grande parte da população mundial, a procura pela reabilitação através do Método Pilates (MP) tem aumentado progressivamete. Objetivos: Realizar uma revisão integrativa sobre os efeitos e benefícios do MP na reabilitação da dor lombar crônica inespecífica. Métodos: As bases de dados utilizadas foram Centro Latino-Americano e do Caribe de Informação em Ciências da Saúde (BIREME) e Physiotherapy Evidence Database (PEDro), Scientific Electronic Library Online (SciELO), foram aplicados os critérios de inclusão e exclusão, após a aplicação destes, foram analisados 9 estudos. Resultados: $O$ presente estudo mostrou que um programa de Pilates para a dor lombar crônica inespecífica repercute positivamente, trazendo como benefícios a diminuição da dor e da incapacidade funcional, bem como aumento da flexibilidade e força muscular. Além disso, melhora a qualidade de vida e diminui o medo de praticar exercício físico. Conclusão: O MP é amplamente utilizado, seus principais benefícios são: diminuição da dor; aumento da capacidade funcional; e melhora da qualidade de vida.

Palavras-chave: Pilates. Dor lombar. Lombalgia
${ }^{1}$ Graduação em Fisioterapia pela Pontificia Universidade Católica de Goiás

E-mail: izabel ca@yahoo.com.br

${ }^{2}$ Graduação em Fisioterapia pela Universidade Estadual de Goiás

\section{ABSTRACT}

Abstract Currently chronic low back pain affects a large part of the world population, the search for rehabilitation through the Pilates Method (MP) has progressively increased. Objectives: To conduct an integrative review of the effects and benefits of PM on the rehabilitation of nonspecific chronic low back pain. Methods: The databases used were the Latin American and Caribbean Center for Health Sciences Information (BIREME) and the Physiotherapy Evidence Database (PEDro), Scientific Electronic Library Online (SciELO), and the inclusion and exclusion criteria were applied after their application, 9 studies were analyzed. Results: The present study showed that a Pilates program for nonspecific chronic low back pain has a positive effect, reducing the pain and functional disability, as well as increasing muscle flexibility and strength. It improves the quality of life and reduces the fear of physical exercise. Conclusion: PM is widely used, its main benefits are: decreased pain; increased functional capacity; and improved quality of life.

Keywords: Pilates. Backache. Low back pain 


\section{INTRODUÇAO}

A dor lombar, devido à sua crescente prevalência, é um desafio para o sistema de saúde e um problema social significativo (ELIKS; ZGORZALEWICZ-STACHOWIAK; ZEŃCZAK-PRAGA, 2019). É considerada uma das queixas musculoesqueléticas mais comuns no mundo, afetando mais pessoas do sexo feminino. Além disso, é a causa número um de incapacidade e de afastamentos do trabalho (HARTVIGSEN et al., 2018), sendo definida por um sintoma de dor ou desconforto que dura pelo menos 12 semanas que pode ser acompanhada ou não de irradiação para os membros inferiores (KANAS et al., 2018).

Esse tipo de lombalgia ocorre sem alterações estruturais, ou seja, não há redução do espaço do disco, compressão de raízes nervosas, lesão óssea ou articular, escoliose ou lordose acentuada que possam levar a dor na coluna (LIZIER; PEREZ; SAKATA, 2012). A natureza inespecífica do sintoma pode estar relacionada a uma fonte músculoligamentar, que quando associada a fatores de qualidade de vida, psicológicos ou físicos produzem o sintoma indesejado (KANAS et al., 2018), dessa forma, sua causa é considerada multifatorial.

Está estabelecido na literatura que o tratamento baseado em exercícios para a dor lombar crônica é eficaz, sendo que os exercícios terapêuticos são provavelmente o tratamento conservativo mais usado e recomendado no mundo todo. No entanto, não há consenso sobre o melhor tipo de exercício a ser realizado, bem como, não há um protocolo especifico (JONES, 2016; LIZIER; PEREZ; SAKATA, 2012).

Diversos tratamentos são usados para controle e tratamento da dor lombar. O Método Pilates (MP) tem se difundido rapidamente sendo muito apreciado pelos pacientes/praticantes. Sua aplicabilidade é diversa, atualmente é utilizado para promoção de saúde, prevenção e tratamento de doenças, principalmente osteomioarticulares, entre elas a dor lombar. O MP foi originalmente desenvolvido por Joseph Pilates durante a Primeira Guerra Mundial e trouxe uma nova visão para os métodos de reabilitação da coluna lombar (POSADZKI; LIZIS; HAGNER-DERENGOWSKA, 2011).

O MP estimula a estabilização segmentar lombo-pélvica priorizando a automatização de padrões de recrutamento muscular, melhora o condicionamento dos músculos do tronco relacionados com a manutenção da estabilidade da região lombar. Exercícios terapêuticos que promovam a estabilização segmentar lombo-pélvica são indicados na fisioterapia para a prevenção e reabilitação da dor lombar crônica 
inespecífica, são caracterizados por isometria, baixa intensidade e ativação sincronizada dos músculos profundos e estilizadores do tronco (SILVEIRA et al., 2018).

Muitas pessoas têm procurado o MP em busca da reabilitação. Na literatura, é possível encontrar estudos que investigaram os benefícios do MP nessa população, no entanto, diante da quantidade de artigos se faz necessário uma sintetize na busca por sistematizar as informações acerca dos efeitos do MP na dor lombar crônica inespecífica. Portanto, o objetivo geral deste estudo foi investigar os efeitos e benefícios do MP no tratamento da dor lombar crônica através de uma revisão bibliográfica.

\section{MATERIAIS E METODOS}

Utilizou-se o método integrativo de revisão de literatura, visando reunir e sintetizar os conhecimentos científicos produzidos sobre o tema em estudo. Isso permitiu a busca, avaliação e síntese das evidências científicas disponíveis para facilitar o desenvolvimento de conclusões sobre o tema. Seis etapas foram seguidas para a elaboração deste estudo: estabelecendo a questão de pesquisa e os objetivos do estudo; definição dos critérios de inclusão e exclusão das publicações; procura literária; análise e categorização de estudos; e apresentação e discussão de resultados. (MENDES; SILVEIRA; GALVÃO, 2008)

A busca foi realizada em agosto de 2019 utilizando as seguintes bases de dados eletrônicas: Centro Latino-Americano e do Caribe de Informação em Ciências da Saúde (BIREME) e Physiotherapy Evidence Database (PEDro), Scientific Electronic Library Online (SciELO). As palavras-chave utilizadas foram: pilates; dor lombar; e lombalgia.

Os critérios de inclusão foram: artigos publicados em revistas indexadas; trabalhos escritos em português ou inglês publicados no período de 2014 a 2019, abordando como forma de reabilitação o MP para a dor lombar crônica inespecífica.

Os critérios de exclusão foram: Trabalhos que abordam distúrbios diferentes daqueles em estudo, relatando apenas o tratamento da dor lombar aguda, ou mencionando tratamentos cirúrgicos ou farmacológicos; e artigos em um idioma diferente do inglês, português.

Foi desenvolvido um instrumento de coleta de informações para responder à questão de pesquisa desta revisão, composto pelos seguintes itens: título, autor, método, periódico, ano de publicação, objetivo do estudo e principais resultados. Após a leitura dos artigos selecionados, foi realizada uma análise e organização dos seguintes temas: 
controle da dor; flexibilidade; equilíbrio; capacidade funcional; qualidade de vida. A análise, categorização e síntese dos temas foi realizada com o objetivo de obter a melhor descrição e classificação dos resultados (MENDES; SILVEIRA; GALVÃO, 2008).

\section{RESULTADOS}

A busca de dados encontrou 134 artigos, os resumos foram lidos e avaliados, destes apenas 9 artigos, todos em inglês, atenderam aos critérios estabelecidos, e dessa forma foram selecionados e lidos na íntegra.

Os estudos de forma geral mostraram efeitos positivos para o MP em pacientes com dor lombar crônica não especifica. Os principais benefícios do método apontados pelos estudos foram principalmente sobre o alivio da dor (CRUZ-DÍAZ et al., 2018; CRUZDÍAZ et al., 2015; MACHADO et al., 2017; MIYAMOTO et al., 2018; NATOUR et al., 2015; VALENZA et al., 2017), a maior na capacidade funcional (CRUZ-DÍAZ et al., 2018; CRUZDÍAZ et al., 2015; KOFOTOLIS et al., 2016; MACHADO et al., 2017; MIYAMOTO et al., 2018; NATOUR et al., 2015; VALENZA et al., 2017) principalmente em pessoas idosas, aumento da flexibilidade e mobilidade (CRUZ-DÍAZ et al., 2015; MACHADO et al., 2017; VALENZA et al., 2017), melhora no equilíbrio corporal (CRUZ-DÍAZ et al., 2015; LOPES et al., 2017; VALENZA et al., 2017), aumento da qualidade de vida (KOFOTOLIS et al., 2016; NATOUR et al., 2015), maior resistência muscular (MACHADO et al., 2017), e redução da cinesiofobia (CRUZ-DÍAZ et al., 2018) (tabela1).

Tabela 1. Descrição dos resultados dos estudos incluídos nesta revisão, 2014-2019.

\begin{tabular}{|c|c|c|c|c|c|}
\hline Ano & Autor & Titulo & Método & Objetivo & Resultado \\
\hline 2018 & $\begin{array}{l}\text { Cruz- } \\
\text { Diaz et } \\
\text { al., }\end{array}$ & $\begin{array}{l}\text { The effectiveness of } \\
12 \text { weeks of Pilates } \\
\text { intervention on } \\
\text { disability, pain and } \\
\text { kinesiophobia in } \\
\text { patients with } \\
\text { chronic low back } \\
\text { pain: a randomized } \\
\text { controlled trial. }\end{array}$ & $\begin{array}{l}\text { Ensaio clínico } \\
\text { randomizado. } \\
\text { Intrumentos: Roland } \\
\text { Morris Disability } \\
\text { Questionnaire, escala } \\
\text { visual analógica e } \\
\text { Tampa Scale of } \\
\text { Kinesiophobia. }\end{array}$ & $\begin{array}{l}\text { Avaliar a } \\
\text { efetividade de } 12 \\
\text { semanas de } \\
\text { prática de Pilates } \\
\text { em pacientes com } \\
\text { dor lombar crônica } \\
\text { não específica. }\end{array}$ & $\begin{array}{l}\text { Principais mudanças } \\
\text { na incapacidade e } \\
\text { cinesiofobia foram } \\
\text { observadas em seis } \\
\text { semanas de } \\
\text { intervenção, a dor } \\
\text { apresentou melhores } \\
\text { resultados em } 12 \\
\text { semanas. }\end{array}$ \\
\hline 2018 & $\begin{array}{l}\text { Miyam } \\
\text { oto et } \\
\text { al., }\end{array}$ & $\begin{array}{l}\text { Different doses of } \\
\text { Pilates-based } \\
\text { exercise therapy for } \\
\text { chronic low back } \\
\text { pain: a randomised } \\
\text { controlled trial with } \\
\text { economic } \\
\text { evaluation }\end{array}$ & $\begin{array}{l}\text { Ensaio clínico } \\
\text { randomizado. } \\
\text { Instrumentos: } \\
\text { Escala visual } \\
\text { analógica de dor; } \\
\text { Roland-Morris } \\
\text { Disability }\end{array}$ & $\begin{array}{l}\text { Avaliar a eficácia } \\
\text { e a rentabilidade } \\
\text { da adição de } \\
\text { diferentes doses } \\
\text { de Pilates para } \\
\text { dor lombar crônica } \\
\text { não específica. }\end{array}$ & $\begin{array}{l}\text { Todos os grupos de } \\
\text { Pilates mostraram } \\
\text { melhorias } \\
\text { significativas na dor e } \\
\text { incapacidade } \\
\text { independentemente } \\
\text { da quantidade de } \\
\text { aulas semanais }\end{array}$ \\
\hline
\end{tabular}




\section{Questionnaire,}

\begin{tabular}{lll}
\hline 2017 & Lopes & Immediate \\
et al., & effects of Pilates \\
& based therapeutic \\
& exercise on postural \\
& control of \\
& young individuals \\
& with non-specific \\
& low back pain: A \\
& randomized \\
& controlled \\
& trial
\end{tabular}

Ensaio controlado
aleatório único cego.
Instrumentos:
Questionário
Internacional de
Atividade Física; The
Oswestry O Disability
Index versão 2;
Escala analógica
visual; Star Excursion
Balance Test;
avaliação postural
usando uma
plataforma de força

$2017 \begin{array}{ll}\text { Macha } & \text { Effectiveness of the } \\ \text { do et } & \text { Pilates method for } \\ \text { al., } & \text { individuals with } \\ & \text { nonspecific low } \\ & \text { back pain: clinical } \\ & \text { and } \\ & \text { electromyographic } \\ & \text { aspects. }\end{array}$

2017 Valeza Results of a Pilates et al., exercise program in patients with chronic non-specific low back pain: a randomized controlled trial

\begin{tabular}{|c|c|c|}
\hline $\begin{array}{l}\text { Coorte prospectivo } \\
\text { observacional. } \\
\text { Avaliação: atividade } \\
\text { eletromiográfica, dor, } \\
\text { flexibilidade, } \\
\text { resistência muscular, } \\
\text { qualidade de vida; e } \\
\text { Questionário de } \\
\text { Crencas de Medo- }\end{array}$ & $\begin{array}{l}\text { O objetivo deste } \\
\text { estudo foi verificar } \\
\text { a influência do } \\
\text { Pilates na } \\
\text { ativação muscular } \\
\text { do multifidio } \\
\text { lombar. }\end{array}$ & $\begin{array}{l}\text { Após oito semanas } \\
\text { de treinamento em } \\
\text { Pilates, houve uma } \\
\text { melhora os } \\
\text { parâmetros clínicos } \\
\text { de dor, flexibilidade, } \\
\text { resistência muscular } \\
\text { e incapacidade. }\end{array}$ \\
\hline
\end{tabular}

objetivo deste
estudo foi
determinar os
efeitos imediatos
dos exercícios de
Pilates na
oscilação postural
e no equilíbrio
dinâmico de
jovens com
lombalgia
inespecífica

Os exercícios de Pilates melhoraram imediatamente a postura e o equilíbrio dinâmico em adultos jovens com lombalgia inespecífica.

\section{Ensaio clínico randomizado. Instrumentos: Roland Morris Disability Questionnaire e Oswestry. Escala visual analógica para dor; e testes físicos; \\ Investigar os efeitos de um programa de exercícios de Pilates em pacientes com dor lombar crônica não específica \\ Um programa de exercícios de Pilates de 8 semanas é eficaz na melhora da incapacidade, dor, flexibilidade e equilíbrio em pacientes com dor lombar crônica não específica.}

$\begin{array}{llll}2016 & \text { Kofotol } & \text { Effects of Pilates } & \text { Ensaio clínico } \\ \text { is et } & \text { and trunk } & \text { randomizado. } \\ \text { al., } & \text { strengthening } & \text { Intrumentos: Short } \\ & \text { exercises on health- } & \text { Form-36v2 Health } \\ & \text { related quality of life } & \text { Survey; Roland Morris } \\ & \text { in } & \text { Disability } \\ & \text { women with chronic } & \text { Questionnaire } \\ & \text { low back pain } & \end{array}$

$\begin{array}{ll}\begin{array}{l}\text { O objetivo deste } \\ \text { estudo foi }\end{array} & \begin{array}{l}\text { Os participantes que } \\ \text { comparar os }\end{array} \\ \text { realizaram um } \\ \text { efeitos de um } & \text { potocolo de pilates } \\ \text { programa de } & \text { relataram mas } \\ \text { Pilates e um } & \text { melhorias na } \\ \text { programa de } & \text { capacidade funcional } \\ \text { exercícios de } & \text { qualidade de vida, em } \\ \text { fortalecimento de } & \text { comparação com os } \\ \text { tronco em } & \text { participantes dos } \\ \text { mulheres com } & \text { grupos exercício de } \\ \text { com dor lombar. } & \text { fortalecimento de } \\ & \text { tronco. Os efeitos } \\ & \text { foram mantidos por } \\ & \text { um período de três } \\ & \text { meses após o término } \\ & \text { do programa para o } \\ & \text { grupo Pilates. }\end{array}$




\begin{tabular}{|c|c|c|c|c|c|}
\hline 2015 & $\begin{array}{l}\text { Cruz- } \\
\text { Diaz et } \\
\text { al., }\end{array}$ & $\begin{array}{l}\text { Effects of a six- } \\
\text { week Pilates } \\
\text { intervention on } \\
\text { balance and fear of } \\
\text { fallingin women } \\
\text { aged over } 65 \text { with } \\
\text { chronic low-back } \\
\text { pain: A } \\
\text { randomizedcontrolle } \\
\text { d trial }\end{array}$ & $\begin{array}{l}\text { Ensaio controlado } \\
\text { aleatório único cego. } \\
\text { Instrumentos: The } \\
\text { Falls Efficacy Scale- } \\
\text { International; Timed } \\
\text { Up and Go; Escala } \\
\text { visual analógica de } \\
\text { dor; }\end{array}$ & $\begin{array}{l}\text { O objetivo do } \\
\text { estudo foi avaliar } \\
\text { os efeitos de seis } \\
\text { semanas de } \\
\text { Pilates sobre o } \\
\text { equilíbrio } \\
\text { funcional, medo } \\
\text { de cair e dor em } \\
\text { mulheres com } \\
\text { mais de } 65 \text { anos } \\
\text { de idade com dor } \\
\text { lombar crônica }\end{array}$ & $\begin{array}{l}\text { Após } 6 \text { semanas } \\
\text { apenas o grupo } \\
\text { Pilates apresentou } \\
\text { melhora no medo de } \\
\text { cair, mobilidade } \\
\text { funcional e equilíbrio, } \\
\text { além disso, redução } \\
\text { da dor quando } \\
\text { comparado com o } \\
\text { grupo que realizou } \\
\text { fisioterapia }\end{array}$ \\
\hline 2015 & $\begin{array}{l}\text { Mosta } \\
\text { gi et } \\
\text { al., }\end{array}$ & $\begin{array}{l}\text { Pilates versus } \\
\text { general exercise } \\
\text { effectiveness on } \\
\text { pain and } \\
\text { functionality in non- } \\
\text { specific } \\
\text { chronic low back } \\
\text { pain subjects }\end{array}$ & $\begin{array}{l}\text { Ensaio clínico } \\
\text { randomizado. } \\
\text { Instrumentos; Quebec } \\
\text { Back Pain } \\
\text { Questionnaire; Escala } \\
\text { Visual analógica de } \\
\text { dor; Testes físicos; }\end{array}$ & $\begin{array}{l}\text { Avaliar a eficácia } \\
\text { do método Pilates, } \\
\text { quando } \\
\text { comparado aos } \\
\text { exercícios gerais, }\end{array}$ & $\begin{array}{l}\text { Não houve diferenças } \\
\text { entre o Pilates e } \\
\text { exercícios gerais em } \\
\text { relação à dor e } \\
\text { funcionalidade, mas } \\
\text { exercícios gerais } \\
\text { foram melhores do } \\
\text { que Pilates para } \\
\text { aumentar a } \\
\text { funcionalidade e } \\
\text { flexibilidade. }\end{array}$ \\
\hline 2015 & $\begin{array}{l}\text { Natour } \\
\text { et al., }\end{array}$ & $\begin{array}{l}\text { Pilates improves } \\
\text { pain, function and } \\
\text { quality of life in } \\
\text { patients with } \\
\text { chronic low back } \\
\text { pain: a randomized } \\
\text { controlled trial. }\end{array}$ & $\begin{array}{l}\text { Ensaio clínico } \\
\text { randomizado; } \\
\text { Instrumentos: Escala } \\
\text { visual analógica de } \\
\text { dor; }\end{array}$ & $\begin{array}{l}\text { Avaliar a eficácia } \\
\text { do método pilates } \\
\text { em pacientes com } \\
\text { dor lombar crônica } \\
\text { não específica } \\
\text { (lombalgia). }\end{array}$ & $\begin{array}{l}\text { O método pilates } \\
\text { pode ser utilizado por } \\
\text { pacientes com } \\
\text { lombalgia para } \\
\text { melhorar a dor, } \\
\text { função e aspectos } \\
\text { relacionados à } \\
\text { qualidade de } \\
\text { (capacidade } \\
\text { funcional, dor e } \\
\text { vitalidade). }\end{array}$ \\
\hline
\end{tabular}

Sobre os estudos selecionados para a pesquisa, a maioria realizou ensaios clínicos randomizados (CRUZ-DÍAZ et al., 2018; KOFOTOLIS et al., 2016; MIYAMOTO et al., 2018; MOSTAGI et al., 2015; NATOUR et al., 2015; VALENZA et al., 2017), dois ensaio controlado aleatório único e cego (CRUZ-DÍAZ et al., 2015; LOPES et al., 2017) apenas um estudo de coorte prospectivo observacional (MACHADO et al., 2017).

\section{DISCUSSAOO}

No presente estudo, foi realizada uma revisão integrativa, abordando estudos sobre a utilização do MP na reabilitação da dor lombar crônica inespecífica. A maioria dos estudos trouxeram efeitos positivos do método em relação a redução da incapacidade funcional, a melhora do equilíbrio, e também redução dos relatos de dores dos pacientes. 
Apenas um estudo mostrou resultados controverso sobre o método, apresentando que exercícios gerais trouxeram maior flexibilidade e funcionalidade a população estudada quando comparado ao MP (MOSTAGl et al., 2015). O estudo de Wells et al. (2013) examinou cinco revisões sistemáticas que investigaram a eficácia do exercício de Pilates em pessoas com dor lombar crônica e concluiu que o Pilates não foi eficaz na redução da dor devido ao baixo número de artigos e da qualidade metodológica destes (WELLS et al., 2013).

O MP é utilizado quase sempre quando a dor e instabilidade já estão instaladas no indivíduo. Por isso, tem papel importante no controle a dor, trabalhando exercícios que visam ao equilíbrio entre forças musculares, o aumento de flexibilidade e resistência do corporal (NOGUEIRA et al., 2014). Um estudo mostrou que os efeitos trazidos pelo método foram mantidos por um período de três meses após o término do programa de reabilitação (KOFOTOLIS et al., 2016). Levando em consideração que é um condição crônica, com causa multifatorial, é recomendado que apesar da melhora dos sintomas o MP continue sendo implementado para a promoção da saúde.

\subsection{CONTROLE DA DOR E CAPACIDADE FUNCIONAL}

Todos estudos abordados nessa revisão que investigaram o controle da dor na lombalgia demostraram resultados positivos. O estudo de Miyamoto et al., (2018) foi realizado com 296 pacientes com idades entre 18 e 80 anos com diagnostico confirmado de lombalgia inespecífica que perdurou por mais de 3 meses, os pacientes foram divididos em quatro grupos, o primeiro recebeu um aconselhamento através de folhetos, o segundo intervenção por meio do MP uma vez por semana, ou terceiro duas vezes por semana e o quarto três vezes por semana. Os resultados mostraram que adição do pilates ao aconselhamento, mesmo que seja apenas uma vez na semana é suficiente para melhora da dor e da capacidade funcional. O MP duas vezes por semana se mostrou mais eficaz comparado a vez por semana, e três vezes por semana mostra efeitos semelhantes a duas vezes por semana. Além disso, o exercício de Pilates três vezes por semana é a opção preferida pela população estudada (MIYAMOTO et al., 2018).

Machado et al., (2017) avaliou doze indivíduos de ambos os sexos com lombalgia não específica, foi avaliada à atividade eletromiográfica dos músculos multífidus lombar, transverso abdominal oblíquo interno antes e após um programa de Pilates. Após oito semanas do protocolo estabelecido houve melhora na dor e incapacidade, aumento da 
flexibilidade, controle motor, força de extensão do tronco e resistência muscular dos estabilizadores do tronco (MACHADO et al., 2017). Vasconcellos et al. (2014) realizou uma revisão sistemática com 47 artigos e contatou-se que o desequilíbrio entre os músculos agonistas e antagonistas do tronco, e a ativação ineficaz do transverso abdominal é fator de risco para a dor lombar, além disso, esses resultados podem ser atenuados mediante a utilização do MP como forma de reabilitação (VASCONCELLOS et al., 2014).

Ribeiro, Oliveira e Blois, (2015) realizaram um estudo comparando um grupo em tratamento com cinesioterapia e outro com MP e perceberam que o tratamento com o MP reduziu significativamente a incapacidade funcional (RIBEIRO; BLOIS, 2015). Além disso, Galdino et al. (2015) realizaram uma revisão na qual encontraram resultados positivos relacionados à eficácia e aos benefícios do MP aplicados na dor lombar crônica não específica, agindo principalmente na redução da dor e incapacidade funcional (GALDINO et al., 2015).

\subsection{FLEXIBILIDADE}

Sobre a flexibilidade um estudo mostrou uma melhora significativa na flexibilidade no grupo que realizou o MP. Esse efeito pode ser explicado pela resposta mecânica de tecidos contráteis e não contráteis e pela resposta neurofisiológica. As posições de alongamento alongam lentamente os tecidos moles e ativam o órgão tendinoso de Golgi. O MP é uma combinação de exercícios de alongamento estáticos e dinâmicos que são adequados e seguros para proporcionar uma flexibilidade crescente (VALENZA et al., 2017).

Guimarães et al. (2014) realizaram um estudo experimental com objetivo de verificar o efeito do MP no nível de flexibilidade de quadril e cintura escapular de idosos, notaram que, no decorrer do estudo, idosas que praticaram Pilates tiveram um aumento na flexibilidade, além de prevenir que ela piore com o avanço da idade. Diante disso, a prática do Pilates é aconselhada para esse público (GUIMARÃES et al., 2014).

\subsection{EQUILÍBRIO}

Cruz-Díaz et al. (2015) realizaram um estudo com 103 mulheres com faixa etária maior de 65 anos. Os pacientes foram alocados aleatoriamente, para grupo fisioterapia e MP ou grupo fisioterapia. Os grupos receberam intervenção durante seis semanas, duas 
vezes por semana. Os resultados obtidos sugerem a adição do Pilates à fisioterapia, pois diminui o medo de queda e aumenta o equilíbrio funcional, aspecto que pode estar ligado à dor lombar e ao risco de quedas em mulheres acima de 65 anos (CRUZ-DÍAZ et al., 2015).

Outro estudo, realizado com quarenta e seis adultos jovens com lombalgia inespecífica analisou imediatamente após o uso do MP o equilíbrio estático através de uma plataforma de oscilação postural e o dinâmico por meio de testes funcionais. Por fim, concluiu que o MP melhorara imediatamente a oscilação postural e o equilíbrio dinâmico em adultos jovens (LOPES et al., 2017). Dessa forma, podemos afirmar que independentemente da idade, o MP tem efeitos positivos no equilíbrio. Sendo que é recomendado principalmente em idosos que tendem a uma perca proprioceptiva conforme a progressão da idade.

\subsection{QUALIDADE DE VIDA}

O MP tem sido indicado para diversos tratamentos, como na Osteoporose, um estudo realizado com mulheres pós-menopausa verificou resultados significativo sobre o aumento da densidade mineral óssea, do desempenho físico e da qualidade de vida no grupo que realizou MP (ANGIN; ERDEN; CAN, 2015). Outro estudo, realizado com 101 mulheres com dor lombar crônica inespecifica revelou que em oito semanas do MP melhorou a qualidade de vida significativamente (KOFOTOLIS et al., 2016).

Os benefícios do MP vão além de questões puramente físicas, considerando os princípios básicos do MP, como concentração, coordenação nos movimentos, centralização da produção de força e respiração, o método tem como objetivo uma atuação global do corpo, proporcionando ganho de força, flexibilidade, equilíbrio, correção postural, diminuição da dor e melhora da coordenação motora, favorecendo uma melhor qualidade de vida (COSTA et al., 2018).

Foram consideradas como limitações deste estudo o fato da qualidade metodológica dos artigos não ter sido avaliada; a diversidade de desenhos de pesquisa e de protocolos de intervenção, dificultando a comparação adequada entre os estudos encontrados.

\section{CONSIDERAÇOES FINAIS}


Com esta revisão, verificou-se que o MP pode ser utilizado pelo fisioterapeuta para diversos fins, mostrando-se uma ferramenta importante para diversificar e agregar no tratamento da dor lombar crônica inespecífica. Percebeu-se que um programa de Pilates traz benefícios na diminuição da dor, na redução da incapacidade funcional, no aumento da flexibilidade e força muscular de indivíduos. Além disso, melhora a qualidade de vida e redução do medo de praticar exercício físico. Porém, em alguns momentos, não se apresentou mais eficaz do que outras modalidades de tratamento.

\section{REFERENCIAS}

ANGIN, E.; ERDEN, Z.; CAN, F. The effects of clinical pilates exercises on bone mineral density , physical performance and quality of life of women with postmenopausal osteoporosis. Journal of Back and Musculoskeletal Rehabilitation, v. 28, p. 849-858, 2015.

COSTA, T. R. DA S. et al. Comparação da percepção da qualidade de vida em idosas praticantes e não praticantes do Método Pilates Quality-of-life perception in elderly women who practice and do not practice the Pilates ' method. Cadernos de Saúde Coletiva, n. September, p. 1-9, 2018.

CRUZ-DÍAZ, D. et al. The effectiveness of 12 weeks of Pilates intervention on disability , pain and kinesiophobia in patients with chronic low back pain : a randomized controlled trial. Clinical Rehabilitation, p. 1-9, 2018.

CRUZ-DÍAZ, D. et al. Maturitas Effects of a six-week Pilates intervention on balance and fear of falling in women aged over 65 with chronic low-back pain : A randomized controlled trial. Maturitas, v. 82, n. 4, p. 371-376, 2015.

ELIKS, M.; ZGORZALEWICZ-STACHOWIAK, M.; ZEŃCZAK-PRAGA, K. Application of Pilates-based exercises in the treatment of chronic non-specific low back pain : state of the art. Postgraduate Medical Journal, v. 95, n. 41, p. 41-45, 2019.

GALDINO, I. I. et al. Efeitos do método pilates em pacientes com lombalgia crônica inespecífica: revisão sistemática. Revista Interdisciplinar Ciências e Saúde, 2015.

GUIMARÃES, A. C. DE A. et al. The effect of Pilates method on elderly flexibility.

Fisioterapia em Movimento, v. 27, n. 2, p. 181-188, 2014.

HARTVIGSEN, J. et al. What low back pain is and why we need to pay attention. The Lancet, v. 371, n. 10137, 2018.

JONES, A. M. Pilates para dor lombar. Diagnóstico e tratamento, v. 21, n. 4, p. 190191, 2016.

KANAS, M. et al. Home-based exercise therapy for treating non- specific chronic low back pain. Revista da Associação Médica Brasileira, v. 64, n. 9, p. 824-831, 2018. 
KOFOTOLIS, N. et al. Effects of Pilates and trunk strengthening exercises on healthrelated quality of life in women with chronic low back pain. Journal of Back and Musculoskeletal Rehabilitation, v. 29, p. 649-659, 2016.

LIZIER, D. T.; PEREZ, M. V.; SAKATA, R. K. Exercícios para Tratamento de Lombalgia Inespecífica. Revista Brasileira de Anestesiologia, v. 62, n. 6, p. 838-846, 2012.

LOPES, S. et al. Immediate effects of Pilates based therapeutic exercise on postural control of young individuals with non-speci fi c low back pain : A randomized controlled trial. Complementary Therapies in Medicine, v. 34, p. 104-110, 2017.

MACHADO, P. M. et al. Effectiveness of the Pilates method for individuals with nonspecific low back pain : clinical and electromyographic aspects. Motriz, v. 23, n. 4, p. 1-8, 2017.

MENDES, K. D. S.; SILVEIRA, R. C. DE C. P.; GALVÃO, C. M. Revisão integrativa: método de pesquisa para a incorporação de evidências na saúde e na enfermagem. Texto \& Contexto - Enfermagem, v. 17, n. 4, p. 758-764, 2008.

MIYAMOTO, G. C. et al. Different doses of Pilates-based exercise therapy for chronic low back pain : a randomised controlled trial with economic evaluation. Journal of Sports Medicine, v. 0, p. 1-11, 2018.

MOSTAGI, F. Q. R. C. et al. Pilates versus general exercise effectiveness on pain and functionality in non-specific chronic low back pain subjects. Journal of Bodywork \& Movement Therapies, v. 19, p. 636-645, 2015.

NATOUR, J. et al. Pilates improves pain , function and quality of life in patients with chronic low back pain : a randomized controlled trial. Clinical Rehabilitation, v. 29, n. 1, p. 59-68, 2015.

NOGUEIRA, T. R. B. et al. Efeitos do método pilates nas adaptações neuromusculares e na composição corporal de adultos jovens. Revista Brasileira de Prescrição e Fisiologia do Exercício, v. 8, n. 45, p. 296-303, 2014.

POSADZKI, P.; LIZIS, P.; HAGNER-DERENGOWSKA, M. Complementary Therapies in Clinical Practice Pilates for low back pain : A systematic review. Complementary Therapies in Clinical Practice, v. 17, n. 2, p. 85-89, 2011.

RIBEIRO, I. A.; BLOIS, C. R. Effects of Pilates and Classical Kinesiotherapy on chronic low back pain : a case study. Fisioterapia em Movimento, v. 28, n. 4, p. 759-765, 2015.

SILVEIRA, A. P. DE B. et al. Efeito imediato de uma sessão de treinamento do método Pilates sobre o padrão de cocontração dos músculos estabilizadores do tronco em indivíduos com e sem dor lombar crônica inespecífica. Fisioterapia e pesquisa, v. 25, n. 2, p. 173-181, 2018.

VALENZA, M. C. et al. Results of a Pilates exercise program in patients with chronic nonspecific low back pain : a randomized controlled trial. CLINICAL REHABILITATION Clinical Rehabilitation, v. 31, n. 6, p. 753-760, 2017.

VASCONCELLOS, M. H. O. DE et al. The Pilates ${ }^{\circledR}$ Method in the treatment of lower back pain. Fisioter. Mov, v. 27, n. 3, p. 459-467, 2014. 
WELLS, C. et al. Effectiveness of Pilates exercise in treating people with chronic low back pain : a systematic review of systematic reviews. BMC Medical Research Methodology, v. 13, n. 7, p. 1-12, 2013. 